\title{
Re-evaluation of Protease Activity of Reelin
}

\author{
Takao KoHNo and Mitsuharu HatToRI* \\ Department of Biomedical Science, Graduate School of Pharmaceutical Sciences, Nagoya City University; 3-1 Tanabe- \\ dori, Mizuho-ku, Nagoya, Aichi 467-8603, Japan. \\ Received February 27, 2010; accepted March 4, 2010; published online March 24, 2010
}

\begin{abstract}
Reelin is a very large secreted glycoprotein that is essential for brain formation and function, but the mechanism by which it affects the dynamics and morphology of neuronal cells remains unsolved. One previous study claimed that Reelin has a proteolytic activity against extracellular matrix proteins, which might explain many of the actions of Reelin. Therefore, in this study wild-type Reelin protein and its mutant in which a supposedly critical serine residue was replaced were expressed and tested for their self-degrading and laminin-degrading activities. We found that both of these proteins generated totally the same cleaved fragments and that neither of them is capable of degrading laminin. It is thus likely that Reelin is not a serine protease and is unable to degrade extracellular matrix.
\end{abstract}

Key words Reelin; protease; brain; extracellular matrix

Reelin is a large glycoprotein consisting of 3461 amino acid residues in mouse. ${ }^{1}$ Reelin plays central roles in brain development, such as neuronal cell migration ${ }^{2)}$ and dendritic growth. ${ }^{3,4)}$ Reelin is also implicated in adult brain function and diseases. ${ }^{5)}$ Therefore, it is important to understand how Reelin exerts its biological activity and how it is regulated.

Reelin protein is cleaved at two sites (between Reelin-repeat 2 (RR2) and RR3; N-t site, between RR6 and RR7; C-t site) in vivo and in vitro. ${ }^{6,7)}$ These cleavages give rise to five kinds of specific fragment, as shown in Fig. 1A. The amount of the fragment from the N-terminus to RR2 (NR2, Fig. 1A) is decreased in schizophrenia and mood disorder patients, ${ }^{8)}$ while it was increased in Alzheimer's disease patients. ${ }^{9)}$ It is therefore of physiological significance to investigate the mechanism of Reelin cleavage. Identification of the protease(s) in charge of Reelin cleavage is particularly important. So far two hypotheses have been proposed for the protease. One is that a protease(s) of the metalloprotease family other than Reelin itself cleaves Reelin. ${ }^{6,710)}$ The other is that Reelin is a serine protease and cleaves itself as well as other proteins. ${ }^{11)}$ Most of the recent reports favor the former scenario, but the latter one has not been tested in detail and could even be compatible with the former one. We thus set out to investigate if Reelin has a serine protease activity against itself and an extracellular matrix protein laminin.

\section{MATERIALS AND METHODS}

Antibodies The mouse monoclonal anti-Reelin antibodies G10 and E5 were purchased from Chemicon (Temeula, CA; 1:2000) and from Santa Cruz Biotechnology (Santa Cruz, CA; $1: 500$ ), respectively.

Vector Construction The expression vector $\mathrm{pCrl}^{12)}$ was used to express Reelin wild-type (WT) and as a template for polymerase chain reaction. The expression vector for the Reelin mutant in which serine 1283 was replaced with cysteine (Reelin1283SC) was constructed as follows. A polymerase chain reaction product harboring the point mutation was obtained by using primers CCTTGTACAAGAAGAATGCC and CAAACCGGTCTCCATCACACTTTCC (the underlined codon corresponds to cysteine), and subcloned into pBluescriptII vector (Stratagene, La Jolla, CA, U.S.A.) that had a BsrGI/AgeI fragment from pCrl. After the sequence was confirmed, the mutant fragment was subcloned into $\mathrm{pCrl}$.

Cell Culture and Transfection Human embryonic kidney (HEK) 293T cells were cultured as previously described. ${ }^{10)}$ Transfection was performed with Lipofectamine 2000 (Invitrogen, Carlsbad, CA, U.S.A.) according to the manufacturer's instructions. After $5 \mathrm{~h}$ of transfection, the culture medium was replaced with serum-free Opti-MEM (Invitrogen), and cultured for another $43 \mathrm{~h}$. The supernatants were filtrated through a $0.45 \mu \mathrm{m}$ filter (Millipore, Bedford, MA, U.S.A.). The concentration of Reelin prepared by this procedure is approximately $0.5 \mathrm{~nm} .{ }^{13)}$

Western Blotting Samples were prepared in the sampling buffer $(62.5 \mathrm{~mm}$ Tris, $\mathrm{pH}$ 6.8, 2\% sodium dodecyl sulfate (SDS), $5 \% \beta$-mercaptoethanol, $10 \%$ glycerol, $0.05 \%$ bromophenol blue), separated by SDS-polyacrylamide gel electrophoresis (SDS-PAGE), and transferred onto a polyvinylidene diffuoride membrane (Millipore). The membrane was incubated with the indicated primary antibody and the appropriate horseradish peroxidase-conjugated secondary antibody. Images were captured using a chemiluminescent reagent (GE Healthcare, Piscataway, NJ, U.S.A.) and a LAS4000 system (Fuji, Tokyo, Japan).

Purification of Reelin The filtrated supernatants were applied to a Hitrap Heparin Sepharose HP column chromatograph set up in an AKTA system (GE Healthcare). The column was run at a flow rate of $0.25 \mathrm{ml} / \mathrm{min}$, washed with $150 \mathrm{~mm} \mathrm{NaCl}$ in Tris, pH 7.5 buffer, and eluted with a linear gradient of $\mathrm{NaCl}(150 \mathrm{~mm}$ to $1 \mathrm{M})$.

Laminin Degradation Assay Purified Reelin proteins were incubated with $5 \mu \mathrm{g}$ laminin (Invitrogen) for $0,10,30$, 120 or $960 \mathrm{~min}$ at $37^{\circ} \mathrm{C}$. These samples were separated by SDS-PAGE. Separated gel was stained with silver staining II kit (Wako, Tokyo, Japan).

\section{RESULTS}

Serine1283 Is Dispensable for the Cleavage of Reelin In most serine proteases, the primary sequence surrounding the active serine residues (Gly-X-Ser-X-Gly) is conserved. Reelin contains this motif, GKSDG, at amino acid residues 1281-1285, which is located in RR3 (Fig. 1A). A previous 


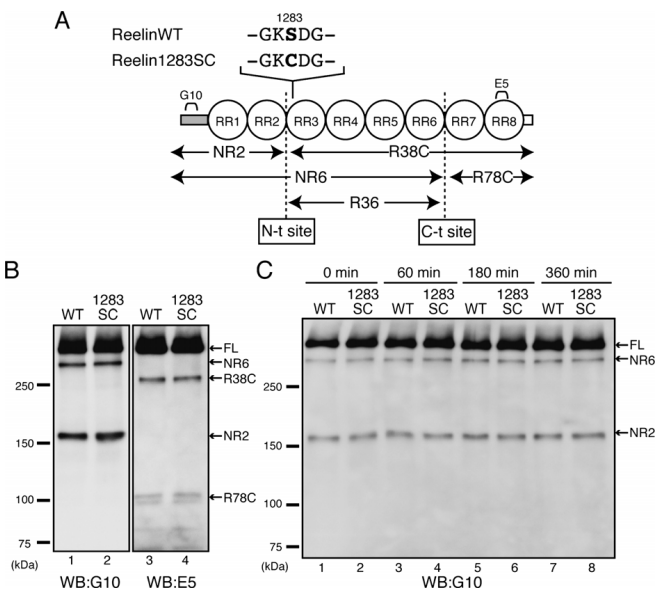

Fig. 1. Serine1283 Is Dispensable for the Cleavage of Reelin

(A) Schematic representation of wild-type (WT) and mutant Reelin. The N-t and C-t cleavage sites are located between RR2 and RR3 and between RR6 and RR7, respectively. RR; Reelin-repeat. The epitopes of G10 and E5 antibodies are shown. (B) ReelinWT or Reelin1283SC was transiently expressed in HEK293T cells. The supernatants were separated by SDS-PAGE, followed by Western blotting using anti-Reelin G10 or E5 antibodies. (C) ReelinWT or Reelin1283SC was incubated at $37^{\circ} \mathrm{C}$ for $0,60,180$ or $360 \mathrm{~min}$. The samples were separated by SDS-PAGE and followed by Western blotting analysis using anti-Reelin G10 antibody. The positions of the molecular weight markers $(\mathrm{kDa})$ are shown on the left of the panels. FL, position of full-length Reelin.

report suggested that this serine residue was labeled with active-serine modifying reagents and that Reelin has a protease activity that had been inhibited by those reagents. ${ }^{11)}$ To confirm this, we constructed the expression vector for Reelin in which the proposed active serine residue (serine1283) is replaced with cysteine (Reelin1283SC, Fig. 1A). ReelinWT or Reelin1283SC was expressed in HEK293T cells and analyzed by Western blotting using anti-Reelin antibodies. AntiN-terminal region antibody G10 detected full-length Reelin (FL), NR6, and NR2 fragments (Fig. 1B, left panel, approximately $430 \mathrm{kDa}, 330 \mathrm{kDa}$, and $160 \mathrm{kDa}$, respectively). AntiRR8 antibody E5 detected FL, R38C, and R78C (Fig. 1B, right panel, $430 \mathrm{kDa}, 270 \mathrm{kDa}$, and $100 \mathrm{kDa}$, respectively). There was no difference in the amount of cleaved fragments between ReelinWT and Reelin1283SC (Fig. 1B). We also investigated whether the incubation of Reelin at $37^{\circ} \mathrm{C}$ promotes its self-cleavage. It was found that self-cleavage of Reelin did not occur at any incubation times $(60,180$, $360 \mathrm{~min}$, respectively), as shown Fig. 1C. These results indicated that the cleavage of Reelin is independent of serine 1283 and that Reelin does not have self-cleaving activity.

Reelin Does not Degrade Laminin Quattrocchi and colleagues $^{11)}$ have previously shown that purified Reelin degraded extracellular matrix proteins, such as fibronectin and laminin, and that the activity was inhibited by active-serine modifiers. We investigated whether the serine 1283 of Reelin is necessary for the degradation of laminin. To prepare the purified Reelin, we separated Reelin from the culture supernatants of Reelin-expressing HEK293T cells by Hitrap Heparin Sepharose column chromatography as Reelin has affinity for heparin. ${ }^{14)}$ ReelinWT and Reelin1283SC proteins were eluted from the column by the buffer containing approximately $450 \mathrm{~mm} \mathrm{NaCl}$, as previously reported. ${ }^{14)}$ In the peak fractions, the full-length protein was exclusively detected by Western blotting, while the cleaved fragment was hardly detected (Fig. 2A). Purified Reelin protein was then incubated with laminin at $37^{\circ} \mathrm{C}$, and its degradation was de-

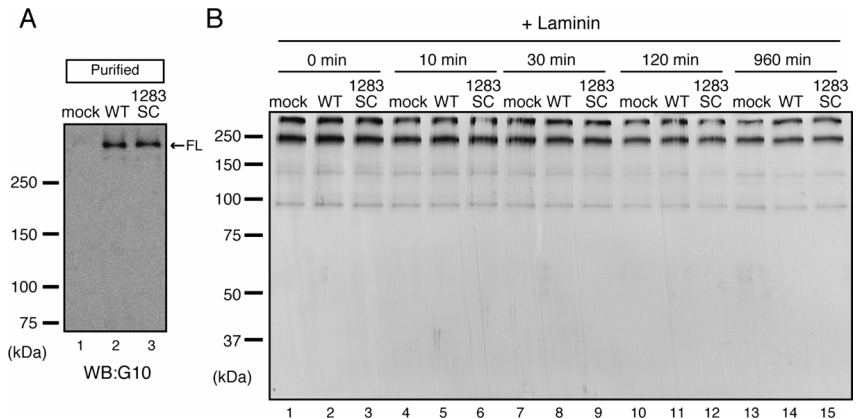

Fig. 2. Reelin Does Not Degrade Laminin

(A) ReelinWT and Reelin1283SC proteins were purified by Hitrap Heparin Sepharose column chromatography. Purified proteins were detected by Western blotting using anti-Reelin G10 antibody. (B) Purified ReelinWT or Reelin1283SC was incubated with laminin at $37^{\circ} \mathrm{C}$ for $0,10,30,120$, or $960 \mathrm{~min}$. The samples were separated by SDS-PAGE and followed by silver staining. The supernatant of HEK293T cells transfected with the empty vector was used as a control. The positions of the molecular weight markers $(\mathrm{kDa})$ are shown on the left of the panels.

tected by silver staining. Contrary to the previous report, ${ }^{11)}$ neither ReelinWT nor Reelin1283SC degraded laminin (Fig. 2B). This result suggested that Reelin does not have serine protease activity for degradation of the extracellular matrix protein.

\section{DISCUSSION}

Although the importance of Reelin in the development and function of mammalian brain is well noted, the molecular mechanism by which it affects cellular function and morphology remains largely unknown. A critical question is whether or not Reelin can act as a protease, since one of the functions of Reelin is to modulate cellular adhesiveness and movement ${ }^{2}$ and the amount of Reelin fragments has been implicated in brain diseases. ${ }^{8,9)}$ In this study, we for the first time introduced a mutation to the proposed active serine residue of Reelin and purified Reelin proteins in order to examine the proteolytic activity of Reelin against itself and laminin. The results clearly showed that fragmentation of Reelin is not affected by serine substitution and that Reelin has no degrading activity against laminin. We speculate that the Reelin sample used in the original study (partially purified by gel filtration and electroelution from the polyacrylamide gel ${ }^{11)}$ might have contained another protease that can degrade Reelin and laminin. It should be also pointed out that serine 1283 is located in RR3 ${ }^{15}$ ) and not in RR2 as was originally suggested. ${ }^{11)}$ In any case, exactly how Reelin transduces signals is still unresolved and needs further study.

Acknowledgements We thank the members of our laboratory for their helpful comments and discussions. This work was supported by the Ministry of Education, Culture, Sports, Science and Technology (KAKENHI 17082007 to M.H. and 08J08604 to T.K.) and by the Research Foundation for Pharmaceutical Sciences. T.K. is a Research Fellow of the Japan Society for the Promotion of Science.

\section{REFERENCES}

1) D’Arcangelo G., Miao G. G., Chen S. C., Soares H. D., Morgan J. I., Curran T., Nature, 374, 719-723 (1995).

2) Tissir F., Goffinet A. M., Nat. Rev. Neurosci., 4, 496-505 (2003). 
3) Niu S., Renfro A., Quattrocchi C. C., Sheldon M., D’Arcangelo G., Neuron, 41, 71-84 (2004).

4) Matsuki T., Pramatarova A., Howell B. W., J. Cell Sci., 121, 18691875 (2008).

5) Herz J., Chen Y., Nat. Rev. Neurosci., 7, 850-859 (2006).

6) Lambert de Rouvroit C., de Bergeyck V., Cortvrindt C., Bar I., Eeckhout Y., Goffinet A. M., Exp. Neurol., 156, 214-217 (1999).

7) Jossin Y., Gui L., Goffinet A. M., J. Neurosci., 27, 4243-4252 (2007).

8) Fatemi S. H., Kroll J. L., Stary J. M., Neuroreport, 12, 3209-3215 (2001).

9) Botella-Lopez A., Burgaya F., Gavin R., Garcia-Ayllon M. S., GomezTortosa E., Pena-Casanova J., Urena J. M., Del Rio J. A., Blesa R., Soriano E., Saez-Valero J., Proc. Natl. Acad. Sci. U.S.A., 103, 5573-
5578 (2006).

10) Kohno S., Kohno T., Nakano Y., Suzuki K., Ishii M., Tagami H., Baba A., Hattori M., Biochem. Biophys. Res. Commun., 380, 93-97 (2009).

11) Quattrocchi C. C., Wannenes F., Persico A. M., Ciafre S. A., D’Arcangelo G., Farace M. G., Keller F., J. Biol. Chem., 277, 303-309 (2002).

12) D’Arcangelo G., Nakajima K., Miyata T., Ogawa M., Mikoshiba K., Curran T., J. Neurosci., 17, 23-31 (1997).

13) Uchida T., Baba A., Pérez-Martínez F. J., Hibi T., Miyata T., Luque J. M., Nakajima K., Hattori M., J. Neurosci., 29, 10653-10662 (2009).

14) Tan K., Duquette M., Liu J. H., Lawler J., Wang J. H., J. Mol. Biol., 381, 1213-1223 (2008).

15) Ichihara H., Jingami H., Toh H., Brain Res. Mol. Brain Res., 97, 190193 (2001). 